\title{
WNT5A gene and protein expression in endometrial cancer
}

\author{
Tomasz Wasniewski ${ }^{1}$, Jacek Kiezun ${ }^{2}$, Bartlomiej E. Krazinski², Anna E. Kowalczyk ${ }^{2}$, \\ Blazej Szostak ${ }^{3}$, Piotr M. Wierzbicki ${ }^{4}$, Jolanta Kiewisz ${ }^{2}$
}

${ }^{1}$ Department of Obstetrics, Perinatology and Gynecology, School of Medicine, Collegium Medicum, University of Warmia and Mazury in Olsztyn, Olsztyn, Poland

${ }^{2}$ Department of Human Histology and Embryology, School of Medicine, Collegium Medicum, University of Warmia and Mazury in Olsztyn, Olsztyn, Poland

${ }^{3}$ Department of Pathomorphology, Regional Specialist Hospital, Olsztyn, Poland

${ }^{4}$ Department of Histology, Medical University of Gdansk, Gdansk, Poland

\begin{abstract}
Introduction. WNT5A (Wnt family member $5 A$ ) belongs to the WNT family of secreted signaling glycoproteins that play essential role in developmental, physiological and pathological processes. WNT5A was shown to take part in carcinogenesis process playing both oncogenic and suppressor functions in various types of human malignancies. This study aimed to assess the expression of the $W N T 5 A$ gene at the mRNA and protein levels in the specimens derived from endometrial cancer (EC) or unchanged control endometrium. The associations between the $W N T 5 A$ expression levels and clinicopathological characteristics and survival of EC patients were evaluated.

Materials and methods. Total RNA was isolated in order to assess the relative amounts of $W N T 5 A$ mRNA by quantitative polymerase chain reaction (QPCR) in samples of unchanged endometrial control $(\mathrm{n}=8)$ and tumor samples of EC patients $(n=28)$. Immunohistochemistry (IHC) was used to determine the presence of WNT5A protein in the sections of formalin-fixed, paraffin-embedded tissue specimens derived from unchanged endometrial controls $(n=6)$ and EC tumors $(n=19)$. Significance of differences in WNT5A expression levels between the studied groups of EC patients and correlations between the WNT5A and demographic data, pathological features, hematological parameters and overall survival of the patients were evaluated by statistical analysis.

Results. The level of $W N T 5 A$ mRNA was decreased in EC in comparison to unchanged endometrium. $W N T 5 A$ expression was associated with primary tumor invasion status exhibiting reduced level of transcripts in EC that involved organs beyond the uterus when compared to the uterus-confined cancers. WNT5A immunoreactivity was visualized in the cytoplasm and nuclei of EC cells as well as in the luminal and glandular epithelial cells of unchanged endometrium. WNT5A mRNA expression levels correlated negatively with cytoplasmic, and positively with nuclear immunoreactivity of the WNT5A protein in the EC cells. In addition, the relationships between blood leucocyte count (in particular granulocytes and lymphocytes) of patients with EC and their WNT5A mRNA and protein expression levels were established. A positive correlation between the nuclear immunoexpression of WNT5A protein in the cancer cells in cell nuclei and mean platelet volume in blood was also found.

Conclusions. The results of the first study of $W N T 5 A$ expression at the transcript and protein levels indicate that it could be considered as a potential marker of molecular changes that take place during EC development. (Folia Histochemica et Cytobiologica 2019, Vol. 57, No. 2, 84-93)
\end{abstract}

Key word: WNT5A expression; endometrial cancer; platelets volume; QPCR; IHC 


\section{Introduction}

Endometrial cancer (EC) is the most common gynecological neoplasm diagnosed in developed countries, the fourth most common cancer in women after breast, lung, and colorectal cancer. Its incidence is increasing [1]. Exposure to endogenous and exogenous estrogens associated with obesity, diabetes, early onset of menarche, nulliparity, late onset of menopause, older age ( $\geq 55$ years) and use of tamoxifen, are considered as the main risk factors leading to EC development $[2,3]$. Patients are often diagnosed when the disease is still limited to the uterus, what results in a good prognosis. Thus, the 5-year overall survival (OS) for EC varies from $74 \%$ to $91 \%$ in non-metastatic patients [4].

Major diagnostic and prognostic problems of EC assessment are often associated with histopathological evaluation. According to WHO classification, seven subtypes of EC can be distinguished [5]. The most common subtype of EC is an endometrial endometrioid adenocarcinoma (EEAC), classified as type I or estrogen-dependent cancer [6]. Endometrial serous adenocarcinoma (ESC) and clear cell endometrial carcinoma (ccEC), aggressive neoplasia carrying a poor prognosis, are estrogen-independent and are classified as Type II [6]. Several research teams have defined immunohistochemical and/or mutation profiles to aid in distinguishing EC subtypes [7-9]. However, classification of EC by histomorphological criteria has a limited reproducibility and is not accurate or precise enough to effectively triage patients into optimal treatment groups. Thus, there is a necessity to develop better diagnostic and prognostic markers that could improve the histopathological examination [10] and would provide more biologically informative data from endometrial tumors that could assist in planning the optimal course of treatment for the affected individuals.

The WNT family consists of 19 secreted glycoproteins which participate in organogenesis during the fetal life as well as in physiological and pathological states postnatally [11]. Intracellular signal transduction pathways regulated by WNT proteins result in cell proliferation, differentiation, migration, adhesion and change of cell polarity [11]. The conservatively preserved WNT5A (Wnt family member $5 A$ ) protein is crucial for prenatal development, and its absence causes intrauterine fetal death associated with developmental defects [12]. Alternative splicing results in the formation of WNT5A protein isoforms [13] which can fulfill both oncogenic and suppressor functions in the process of carcinogenesis [14].
Moreover, WNT5A influences the cancer stem cell population and tumor microenvironment. It is an important molecule for the regulation of cellular senescence, epithelial-mesenchymal transition (EMT), metastasis, tumor cell metabolism and chemotherapy resistance of cancer cells in various cancer types [15]. The expression of the WNT family members, including WNT5A, in EC was evaluated by Bui et al. [16]; however, they analyzed only a very small group of patients with EC. Therefore, the present study was designed and performed to analyze and compare the expression of the $W N T 5 A$ gene in unchanged endometrial control and tumor samples of EC patients at both mRNA and protein levels by the use of quantitative PCR (QPCR) and immunohistochemistry (IHC), respectively. We correlated the obtained data with clinicopathological features of the patients as well as with the OS to assess whether the evaluation of WNT5A gene or protein expression can provide a new diagnostic and/or prognostic marker in EC.

\section{Material and methods}

Patients. After receiving institutional review board approval, patients were recruited at Division of Gynecology and Obstetrics and Gynecology Oncology Center at The Regional Specialist Hospital in Olsztyn, Faculty of Medicine, University of Warmia and Mazury in Olsztyn, Poland. All participants provided written informed consent for tissue and blood sample donation. The control group $(\mathrm{n}=8)$ included women (age: $47 \pm 7.63$, mean $\pm \mathrm{SD}$; BMI 18.5-25, $\mathrm{n}=4$; BMI 25-30, $\mathrm{n}=2$; $\mathrm{BMI}>30$, $\mathrm{n}=2$ ), subjected to diagnostic curettage of the uterus and surgery, in which histopathological examination of EC and endometrial hyperplasia (hyperplasia simplex and complex, without atypia and with atypia) proved to be negative. The EC group $(n=28)$ included women (age: $66.95 \pm 10.38$, mean \pm SD; BMI 18.5-25, $\mathrm{n}=3$; BMI 25-30, $\mathrm{n}=7$; BMI $>30$, $\mathrm{n}=18$ ) with histologically diagnosed EC of the uterus, detected by diagnostic curettage of the uterine cavity and treated with surgery (hysterectomy, salpingo-oophorectomy, cytological peritoneal washing and brushing, and pelvic and aortic lymph node dissection). Diagnosis of uterine cancer and its status were established by means of clinical, laboratory and histopathological evaluation. None of the patients received any anticancer therapy before the surgery. Ongoing acute illnesses (i.e. infection, non-infectious inflammation, cardiovascular events) or disease that had occurred within the last 30 days, known active malignancy, long-term estrogen therapy, polycystic ovarian syndrome, obesity, diabetes mellitus and hypertension as well as drug or alcohol abuse, excluded patients from the study. After the surgery, data on the OS were collected for all patients. 
Material collection and clinical parameters estimation. Blood samples were collected from the fasting patients before surgery. Blood morphology parameters and activated partial thromboplastin time (APTT) were measured using Cobas 6000 multianalyzer (Roche Diagnostics Ltd., Basel, Switzerland).

Tissue samples $(5 \times 5 \times 5 \mathrm{~mm})$ were taken from areas of endometrial tumors without blood-cysts, hemorrhage or necrosis which could disturb further molecular assays. Multiple sample blocks were taken from the same tumor-suspected area to compensate for eventual tumor heterogeneity. The samples were snap-frozen in liquid-nitrogen immediately after excision and stored at $-80^{\circ} \mathrm{C}$ for mRNA isolation and further molecular studies. Tissue sections for routine diagnostic pathomorphological assessment and IHC were fixed in a $10 \%$ neutral-buffered formalin. After $12 \mathrm{~h}$ sections were dehydrated in a graded ethanol series (50-96\%), cleared in xylene and embedded in paraffin for further processing.

Tumor phenotype classification. The criteria of TNM system following American Joint Committee on Cancer (AJCC) were adopted to establish primary tumor status [17]. Patients classified as T1a $(\mathrm{n}=10)$ and T1b $(\mathrm{n}=6), \mathrm{T} 2(\mathrm{n}=7)$ and T3 ( $n=5)$ were considered. Subsequently, the patients were grouped depending on: the depth of myometrium invasion as less or more than halfway ( $\mathrm{n}=12$ and $\mathrm{n}=16$, respectively), uterine cervix infiltration as negative and positive $(n=14$ and $n=14$, respectively) and uterus-limited tumor growth or involvement of other organs by direct extension beyond the uterus ( $n=23$ and $n=5$, respectively). Pathomorphological assessment was based on a routine light microscopy evaluation. Malignancy grade was assessed according to the International Federation of Gynecology and Obstetrics (FIGO) system [18], as low grade $(\mathrm{G} 1 ; \mathrm{n}=2)$, moderate grade $(\mathrm{G} 2 ; \mathrm{n}=19)$, and high grade $(\mathrm{G} 3 ; \mathrm{n}=7)$. Grades 1 and 2 were grouped together (named further G2). The absence $(n=15)$ or presence $(n=13)$ of endometrial fibroids (myomas) were also recorded for each patient.

Total RNA isolation and Real time PCR. Endometrial tissues were homogenized using MagNA Lyser Instrument and MagNA Lyser Green Beads (Roche Molecular Systems, Inc., Pleasanton, CA, USA). Extraction of total RNA was conducted using mirVana Isolation Kit (Ambion; Thermo Fisher Scientific, Inc., Waltham, MA, USA), according to the protocol provided by manufacturers. The quality and quantity of isolated RNA was estimated with NanoDrop 1000 spectrophotometer (Thermo Fisher Scientific, Inc.). Obtained RNA samples were stored at $-80^{\circ} \mathrm{C}$ until further analysis. The reverse transcription was conducted according to the protocol of HighCapacity cDNA Reverse Transcription Kit (Applied Biosystem; Thermo Fisher Scientific, Inc., Waltham, MA, USA). Quantification of WNT5A and peptidylprolyl isomerase A (PPIA) cDNA in collected samples
(Hs00998537_m1 and Hs99999904_m1) was carried out using ABI 7500 Fast Real-Time PCR System and TaqMan ${ }^{\circledR}$ Fast Advanced Master Mix (all: Applied Biosystem; Thermo Fisher Scientific, Inc.) according to the manufacturer's instructions, and using the following conditions: polymerase activation for $20 \mathrm{sec}$ at $95^{\circ} \mathrm{C}$, followed by 40 cycles of denaturation at $95^{\circ} \mathrm{C}$ for $3 \mathrm{sec}$ and annealing/extension at $60^{\circ} \mathrm{C}$ for $30 \mathrm{sec}$. All samples were quantified in duplicate. No template control reactions were performed for each QPCR run. Standard curves consisting of serial dilutions of the appropriate cDNA were used to control the efficiency of QPCR. Relative quantification of WNT5A expression was evaluated using the ddCt method [19]. Quantified gene had the $\mathrm{Ct}$ normalized against PPIA expression $(\mathrm{dCt})$ as the reference [20].

Immunohistochemistry. Formalin-fixed, paraffin-embedded blocks of unchanged (control) endometrium obtained from 6 patients without EC, and fragments of tumor samples from 19 patients with EC, were cut into $5 \mu \mathrm{m}$-thick sections, soaked in xylene and rehydrated in a series of decreasing ethanol dilutions (96-50\%). Antigen retrieval was carried out using Epitope Retrieval Solution ( $\mathrm{pH}$ 6; Dako; Agilent Technologies Inc., Santa Clara, CA, USA), microwaving for $12 \mathrm{~min}$ at $97^{\circ} \mathrm{C}$. Endogenous peroxide activity was blocked in a solution of $3 \%$ hydrogen peroxide in methanol for $10 \mathrm{~min}$ at room temperature (RT). Tissue sections were incubated with $2.5 \%$ normal horse serum (NHS; Vector Laboratories; Maravai Life Sciences, Inc., Chicago, IL, USA) in buffer containing $0.1 \mathrm{MPBS}$ and $3 \%$ bovine serum albumin (BSA; Sigma-Aldrich; Merck, KGaA, Darmstadt, Germany) for $30 \mathrm{~min}$ at RT. Rabbit anti-human WNT5A (H-58) antibody (sc-30224; Santa Cruz Biotechnology Inc., Dallas, TX, USA), were diluted in NGS/PBS/BSA buffer (1:100). After overnight incubation at $4^{\circ} \mathrm{C}$ with primary antibody, slides were washed 3 times with PBS containing Tween20 (Sigma-Aldrich; Merck, kGa) and incubated for 30 min at RT with goat anti-rabbit antibody diluted according to the protocol provided by manufacturers (ImmPRESS Universal reagent Anti-Mouse/Rabbit Ig; Vector Laboratories; Maravai Life Sciences, Inc.). Reaction was visualized after short incubation with 3,3'-diaminobenzidine (DAB; Liquid DAB + Substrate Chromogen System; Dako; Agilent Technologies Inc.). After counterstaining in Mayer's hematoxylin (Sigma-Aldrich; Merck, KGaA), dehydration in series of alcohol dilutions (50-96\%) and mounting in DPX medium (Sigma-Aldrich; Merck, KGaA) slides were vied using XC-50 camera mounted on Olympus BX-41 (Olympus Corporation, Tokyo, Japan) light microscope by a pathologist who was blinded to the patients' clinical data. Pictures were taken with Panoramic Digital Slide Scanner MIDI (3DHistech Ltd., Budapest, Hungary). Negative controls were obtained by incubation of sections without primary antibody. 
The estimation of WNT5A staining intensity and heterogeneity. The classification of intensity and heterogeneity of the staining were conducted according to the IRS (immunoreactivity score) scale as described by Remmele and Stegner [21]. Briefly, the staining intensity was estimated as 1 (the weakest), 2 (moderate), or 3 (the most intense). Heterogeneity characterized the proportion of positively stained tumor cells was evaluated as 0 points, absence of cells with positive reaction; 1 point, $1-10 \% ; 2$ points, $1-50 \%$; 3 points, $51-80 \%$; 4 points, $>80 \%$ cells with positive reaction by estimation on screening wide areas within each tissue section. The obtained values of intensity and heterogeneity were multiplied and scored from 0 to 12 points. All cases with negative immunoreactivity were scored as 0 .

Statistical analysis. All statistical analyses were conducted using GraphPad PRISM v. 6.0 software (GraphPad Software, Inc., San Diego, CA, USA). Comparisons of $W N T 5 A$ mRNA and immunoreactivity levels in unchanged and endometrial tumor samples were conducted with Mann-Whitney tests. Statistical differences of mRNA levels between EC tissue groups in respect to: primary tumor status, tumor myometrial invasion, infiltration of the tumor on the cervix, invasion beyond the uterus, malignancy grade and presence of endometrial fibroids (myomas) were examined using Kruskal-Wallis and Mann-Whitney tests. All correlations of WNT5A gene and protein expression in both studied patient groups were calculated using a Spearman test. Survival curves were plotted according to the Kaplan-Meier method and the significance of differences in OS between groups of patients was evaluated by the log-rank test. Differences were considered to be statistically significant at $\mathrm{p}$ value less than 0.05 .

\section{Results}

\section{Characteristics of the patients}

Demographic data and blood parameters of control and EC patients included in the study are presented in Table 1.

\section{WNT5A expression at the mRNA level in EC}

In both control and EC samples, the presence of WNT5A mRNA was observed. Samples with $W N T 5 A$ expression ratio below 1.0 were considered as down-regulated and those with ratio above $1.0 \mathrm{us}$ up-regulated. The $W N T 5 A$ transcript level was decreased in $24 / 28(85.72 \%)$ and was elevated in $4 / 28$ $(14.28 \%)$ patients with EC. The mean $W N T 5 A$ gene expression was reduced in EC patients compared to control group ( $\mathrm{p}<0.0001$; Fig. 1A).

WNT5A mRNA levels were significantly lower in the group of patients characterized by tumor invasion beyond the uterus when compared to the uter-
Table 1. Demographic data and blood parameters of the studied cohort of patients

\begin{tabular}{|l|c|c|c|}
\hline & Units & Control & Endometrial cancer \\
\hline $\mathrm{N}$ & & 8 & 28 \\
\hline Age & years & $47 \pm 7.63$ & $66.95 \pm 10.38$ \\
\hline $\mathrm{BMI}$ & $\mathrm{kg} / \mathrm{m}$ & $27.88 \pm 5.92$ & $31.39 \pm 5.47$ \\
\hline $\mathrm{RBC}$ & $10^{6} / \mu \mathrm{l}$ & $4.57 \pm 0.50$ & $4.77 \pm 0.45$ \\
\hline $\mathrm{Hb}$ & $\mathrm{g} / \mathrm{dl}$ & $12.16 \pm 1.89$ & $13.86 \pm 1.36$ \\
\hline $\mathrm{Ht}$ & $\%$ & $38.28 \pm 4.63$ & $41.88 \pm 3.17$ \\
\hline WBC & $10^{3} / \mu \mathrm{l}$ & $7.18 \pm 1.60$ & $7.36 \pm 2.14$ \\
\hline Granulocytes & $\%$ & $63.59 \pm 7.10$ & $60.32 \pm 8.74$ \\
\hline Neutrophils & $\%$ & $61.34 \pm 8.08$ & $57.95 \pm 9.26$ \\
\hline Eosinophils & $\%$ & $1.6 \pm 0.73$ & $1.94 \pm 1.28$ \\
\hline Basophils & $\%$ & $0.52 \pm 0.26$ & $0.43 \pm 0.26$ \\
\hline Lymphocytes & $\%$ & $28.46 \pm 6.07$ & $30.46 \pm 7.83$ \\
\hline Monocytes & $\%$ & $5.79 \pm 1.06$ & $6.49 \pm 1.81$ \\
\hline Platelets & $10^{3} / \mu 1$ & $296.4 \pm 78.52$ & $229.9 \pm 60.26$ \\
\hline MPV & $\mathrm{fl}$ & $10.5 \pm 0.96$ & $10.93 \pm 0.78$ \\
\hline APTT & $\mathrm{s}$ & $26.47 \pm 1.75$ & $27.49 \pm 3.61$ \\
\hline
\end{tabular}

The results are presented as means \pm SD. Abbreviations: BMI - Body Mass Index; RBC - Red Blood Cells; Hb - Hemoglobin; $\mathrm{Ht}-\mathrm{He}-$ matocrit; WBC - White Blood Cells; MPV - Mean Platelet Volume; APTT - Activated Partial Thromboplastin Time

us-confined EC samples ( $\mathrm{p}<0.05$; Fig. 1E). WNT5A expression also tended to correlate with the depth of myometrial invasion exhibiting lower transcript level in tumors that have grown deeper than halfway through the myometrium as compared to those which invaded less than the half of myometrium thickness, but this difference was not confirmed by the statistics $(p=0.06$; Fig. 1C). There were no statistically significant associations between the WNT5A mRNA levels and primary tumor status (established as T1a, T1b, T2 and T3; Fig. 1B), infiltration of the tumor on the cervix (absent or present; Fig. 1D), malignancy grade (G2 and G3; Fig. 1F) and occurrence of uterine fibroids (absent or present; Fig. 1G).

\section{The immunoreactivity of the WNT5A protein in control and EC tissue}

Tissue samples of 6 control and 19 EC patients previously analyzed for their $W N T 5 A$ mRNA expression levels were subjected to IHC in order to assess the immunoexpression of WNT5A protein in the normal endometrium and EC cancer cells.

Representative microphotographs of IHC presenting the location of WNT5A protein in the control endometrium and tumor tissue of EC patients are 


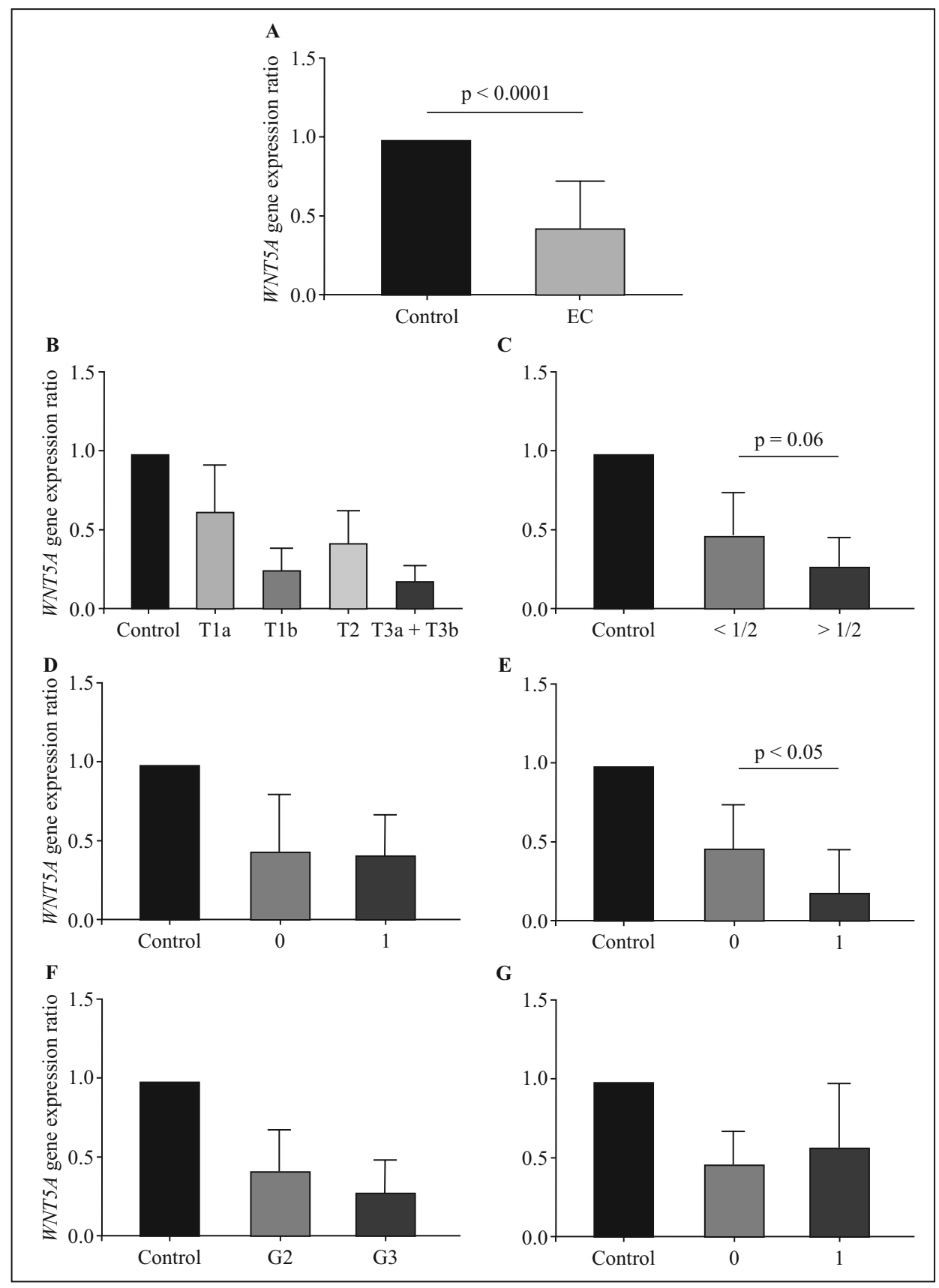

Figure 1. Quantitative Real-time PCR analysis was used to compare $W N T 5 A$ gene expression in tumor tissue of patients with endometrial cancer (EC) and normal endometrial tissue (control). WNT5A mRNA levels in control endometrial samples $(\mathrm{n}=8)$ and EC samples $(\mathrm{n}=28)(\mathbf{A})$. Comparison of the WNT5A mRNA level in a control endometrial samples and samples of EC patients depending on the primary tumor status $(\mathbf{B})$, tumor myometrial invasion $(\mathbf{C})$, absence $(0)$ or presence $(1)$ of tumor infiltration on the cervix $(\mathbf{D})$, presence at the area or beyond the uterus $(\mathbf{E})$, grade $(\mathbf{F})$, absence $(0)$ or presence $(1)$ of endometrial fibroids (G). Gene expression data were normalized against PPIA mRNA levels and presented as $W N T 5 A$ gene expression ratios $\pm \mathrm{SD}$ as described in Methods.

shown in Figure 2. The figures presenting negative control samples were not shown.

The histologically-unchanged endometrial fragments showed dominant, strong, homogenous positive WNT5A immunoreactivity in the cytoplasm and, sporadically, in the nuclei of epithelial cells, whereas in tumor tissue, WNT5A protein staining were mainly present in the nuclei of cancer cells. When control and EC tissue sections were compared, qualitative differences between the immunoreactivity in cytoplasmic and nuclear compartments were observed (Fig. 2). However, there were no quantitative alterations of 

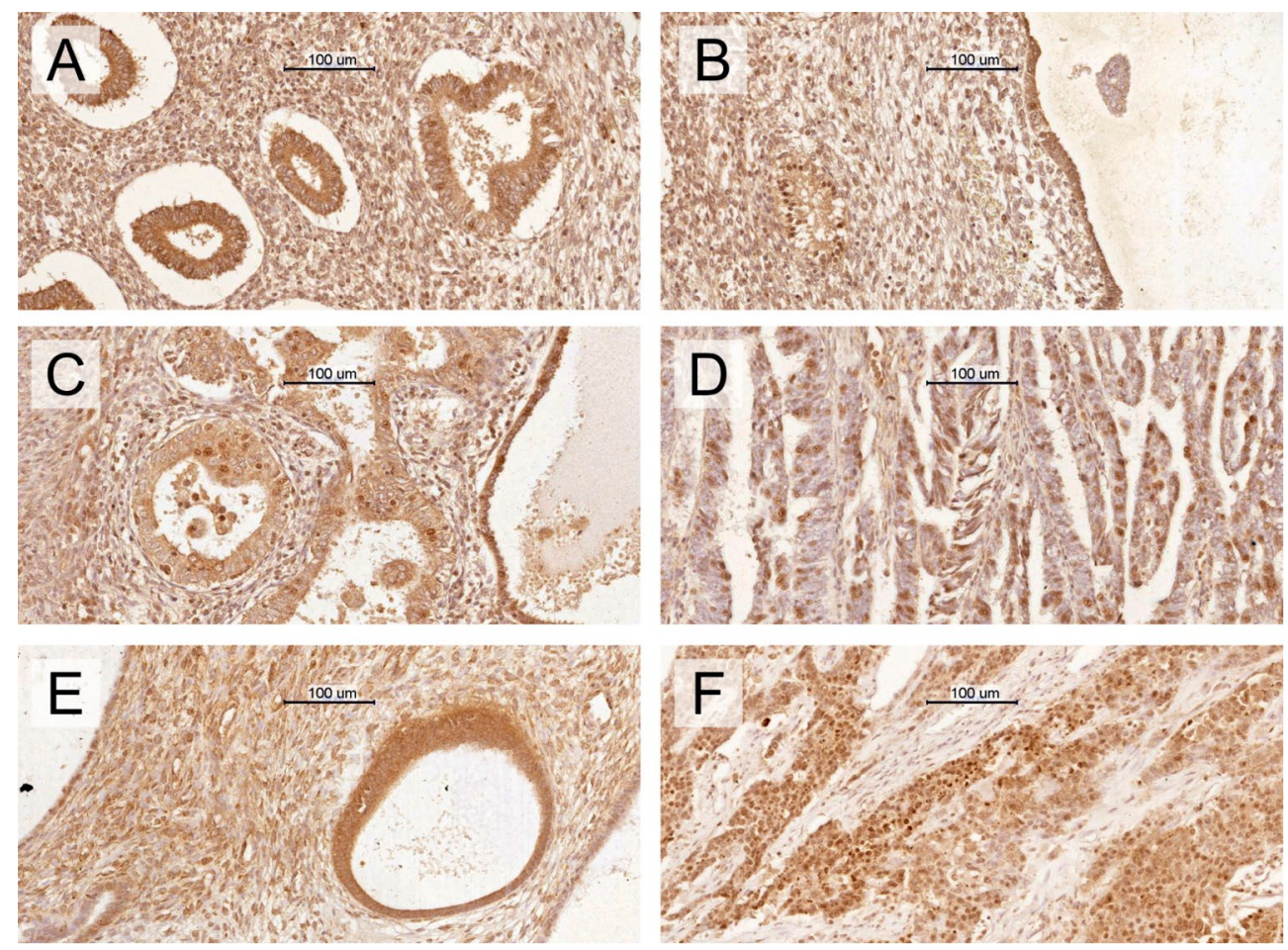

Figure 2. Representative examples of $W N T 5 A$ protein immunoreactivity in normal endometrium (A, B) and endometrial cancer (EC) samples depending on the degree of histological differentiation; endometrium at grade 2 (C, D) and grade 3 (E, F) of EC progression. The immunohistochemical reactions were performed as described in Methods.

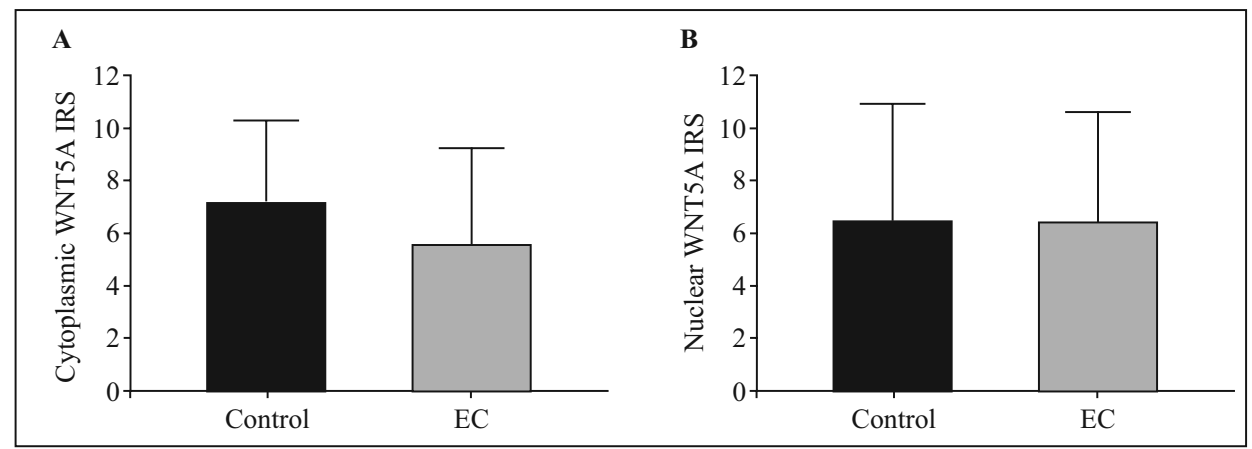

Figure 3. Quantitative analysis of the immunoreactivity of WNT5A protein in cytoplasmic (A) and nuclear (B) compartments of control endometrium $(n=6)$ and endometrial cancer $(E C)$ samples $(n=19)$. The immunoreactivity score (IRS) values are shown as mean \pm SD.

WNT5A immunoreactivity in cytoplasm and nuclear compartments between the unchanged endometrium and EC samples ( $p>0.05$; Fig. 3 A and B, respectively).

WNT5A protein immunoexpression measured on the basis of the IRS scale in the cytoplasmic and nuclear compartments of EC cells did not correlate with clinicopathological characteristics of the patients (data not showed).

\section{Correlations between WNT5A mRNA levels and WNT5A protein immunoreactivity in the cytoplasm and nuclei of tumor cells}

A negative correlation was observed between $W N T 5 A$ mRNA expression and protein immunoexpression in the cytoplasm of EC cells (Rho $=-0.55 ; \mathrm{p}<0.05)$. In contrast, a positive correlation was observed between the $W N T 5 A$ transcript levels in EC tumor samples and 
WNT5A immunoreactivity in the nuclei of cancer cells $($ Rho $=0.60 ; \mathrm{p}<0.01)$.

\section{Correlations between blood parameters and WNT5A $\mathrm{mRNA}$ expression levels and protein immunoreactivity in EC specimens}

In patients with EC, $W N T 5 A$ gene expression correlated positively with the count of leukocytes (WBCs; $\mathrm{p}<0.05)$ and the percentage of granulocytes $(p<0.05)$, and negatively with the percentage of lymphocytes in blood $(\mathrm{p}<0.5$; Table 2$)$.

In patients with $\mathrm{EC}$, we observed negative correlation between the cytoplasmicimmunoreactivity of the WNT5A protein and percentage of granulocytes $(p<0.01)$ or neutrophils $(\mathrm{p}<0.01)$ in blood when the protein in the cytoplasm of EC cells was concerned (Table 2). The percentage of lymphocytes in blood positively correlated with the immunoreactivity of WNT5A in the cytoplasm of EC cells ( $p<0.01$; Table 2). Mean platelet volume (MPV) was the only blood parameter which positively correlated with WNT5A protein immunoreactivity in the nuclei of EC cells $(p<0.05)$. No correlations with age, BMI as well as or other estimated blood parameters such as RBC, WBC and platelets counts, types of leukocytes, hemoglobin $(\mathrm{Hb})$, hematocrit $(\mathrm{Ht}), \mathrm{MPV}$ and APTT, were found when nuclear localization of WNT5A was considered in EC patients (Table 2).

\section{Correlations between WNT5A gene and protein expression and $O S$ of patients with $E C$}

The evaluation of the $W N T 5 A$ gene expression level as a prognostic factor in the studied group of the patients with EC was assessed after the follow up of patients $(n=28)$ and the median observation period was 59 months. During the observation period 25\% $(\mathrm{n}=7)$ patients died. We found a significant correlation between the tumor grade and OS of the patients ( $p<0.001$; Fig. 4A).

In order to investigate associations between OS and $W N T 5 A$ expression EC specimens were divided into the respective cohorts. Based on the median $W N T 5 A$ mRNA relative expression $(\mathrm{RQ}=0.495)$ the patients were divided into two groups regarded as $<0.495(\mathrm{n}=14)$ and $>0.495(\mathrm{n}=14)$. Consequently, the patients were dichotomously divided on the basis of their median value for the cytoplasmic (IRS $=4$ ) or nuclear (IRS = 6) WNT5A immunoreactivity score. We found that the expression level of $W N T 5 A$ mRNA (Fig. 4B) as well as the intensity of cytoplasmic or nuclear WNT5A immunoreactivity (Fig. 4C and D, respectively) did not correlate with patients' OS.

\section{Discussion}

There is an urgent need for intense research in the field of molecular biology of endometrial tumors in or-
Table 2. Correlations between blood parameters and $W N T 5 A$ mRNA levels and WNT5A protein immunoreactivity in cytoplasmic and nuclear compartments of endometrial cancer cells

\begin{tabular}{|l|c|c|c|}
\hline & \multirow{2}{*}{$\begin{array}{c}\text { WNT5A } \\
\text { mRNA }\end{array}$} & \multicolumn{2}{|c|}{ WNT5A protein } \\
\cline { 3 - 4 } & & Cytoplasm & Nucleus \\
\hline Age & -0.22 & 0.18 & -0.16 \\
\hline BMI & 0.31 & -0.39 & 0.23 \\
\hline RBC & -0.01 & -0.19 & -0.08 \\
\hline Hb & -0.15 & 0.02 & -0.19 \\
\hline Ht & 0.24 & 0.05 & -0.13 \\
\hline WBC & $\mathbf{0 . 3 9}$ & -0.36 & 0.15 \\
\hline Granulocytes & $\mathbf{0 . 4 2 *}$ & $-\mathbf{0 . 6 6} * *$ & 0.24 \\
\hline Neutrophils & 0.32 & $-\mathbf{0 . 6 1} * *$ & 0.30 \\
\hline Eosinophils & -0.03 & -0.04 & -0.32 \\
\hline Basophils & 0.18 & 0.12 & -0.35 \\
\hline Lymphocytes & $\mathbf{- 0 . 3 8 *}$ & $\mathbf{0 . 6 7 * *}$ & -0.20 \\
\hline Monocytes & -0.09 & 0.22 & 0.005 \\
\hline Platelets & 0.10 & 0.006 & -0.30 \\
\hline MPV & 0.30 & -0.07 & $\mathbf{0 . 5 1} *$ \\
\hline APTT & -0.09 & -0.18 & 0.29 \\
\hline
\end{tabular}

Abbreviations as in the legend to Table 1. Asterisks indicate statistical significance; ${ }^{*} \mathrm{p}<0.5 ; * * \mathrm{p}<0.01$ ).

der to detect the molecular markers of carcinogenesis process. The present study is the first that used both QPCR and IHC techniques to establish WNT5A expression at the transcript and protein levels in the EC tissue. Our study demonstrated that WNT5A mRNA is down-regulated in the tumor samples as compared to normal endometrial tissue fragments. These findings support the study of Bui et al. [16] who investigated the expression of seven genes belonging to the WNT family in neoplastically-altered and normal endometrium by ribonuclease protection assay. Their results suggested reduced level of $W N T 5 A$ expression in EC tumor tissue and human EC cell lines [16]. Unfortunately, the study of Bui et al. involved only four patients with EC and 11 control cases and its findings were neither supported by statistical analysis nor the correlations between $W N T 5 A$ expression and clinicopathological parameters [16]. Therefore, our research was carried out on a larger cohort of patients and is the first study which shows associations between the reduced $W N T 5 A$ expression and increasing invasive properties of EC tumors.

In our cohort of the patients with EC the WNT5A gene expression was markedly reduced in the tumors that spread outside the uterus as compared to the organ-confined cases. Our results suggest that 


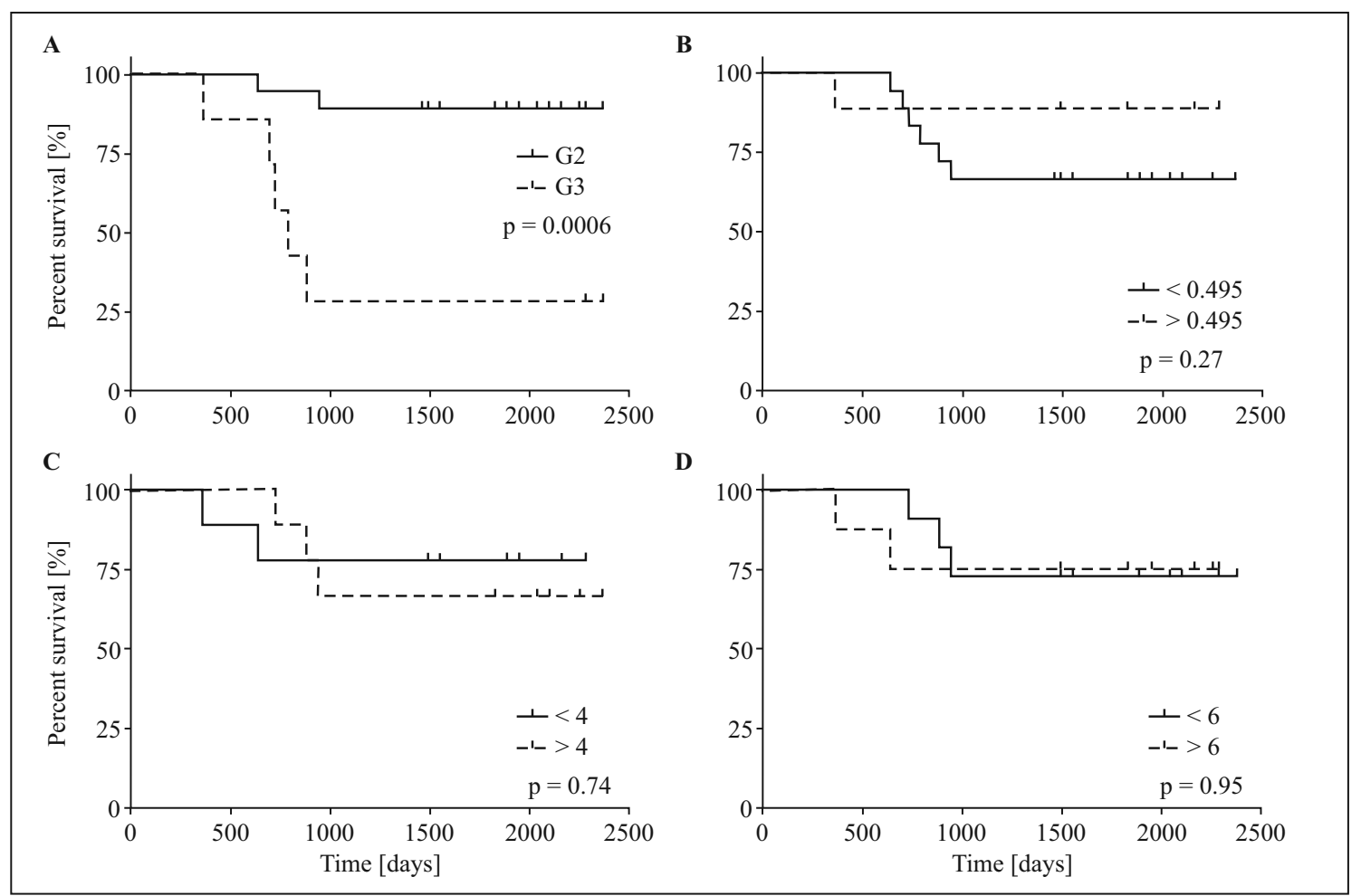

Figure 4. Analysis of the overall survival (OS) of patients with endometrial cancer (EC). Kaplan-Meier survival curves of EC patients relating to $(\mathbf{A})$ the histological grade of EC tumor $(\mathrm{n}=28),(\mathbf{B})$ levels of $W N T 5 A$ mRNA $(\mathrm{n}=28),(\mathbf{C})$ cytoplasmic $(\mathrm{n}=19)$ and $(\mathbf{D})$ nuclear $(\mathrm{n}=19)$ immunoreactivity of the WNT5A protein. P-values of log-rank test are shown.

decreased WNT5A mRNA level could also correlate with the depth of invasion through myometrium. Although these findings could suggest tumor suppressor role of WNT5A in EC, we did not disclose any significant relationship between its expression levels and overall survival of the patients. Indeed, the precise status of the $W N T 5 A$ gene as a factor that might have influenced cancer progression has not been established yet and the opposing observations in other human tumors imply that role of WNT5A protein could be tissue- and/or cancer-specific [14]. In the female reproductive system the decreased expression of $W N T 5 A$ was documented in epithelial ovarian cancer and this reduction was attributed to the hypermethylation of the gene promoter [22]. In contrast to the latter study, Peng et al. found that WNT5A immunoreactivity was increased in the ovarian cancer cells as compared to benign tumors and normal ovarian tissue [23]. Furthermore, elevated level of WNT5A protein was associated with shorter overall and progression-free survival of the patients with ovarian cancer [23]. Also in patients with cervical cancer $W N T 5 A$ expression was found to be either decreased [13] or increased [24]. Using QPCR and IHC techniques Lin et al. [24] showed that the expression of $W N T 5 A$ mRNA was up-regulated in the primary cervical tumors and WNT5A immunoreactivity in cancer cells correlated with the occurrence of distant metastases and worse prognosis. These discrepancies may be partially explained by the findings of Bauer $e t$ al. [13] who demonstrated that the tumor-promoting or tumor-suppressing role of WNT5A in cervical cancer can be attributed to the differential expression of two WNT5A isoforms that have distinct effects on cancer-relevant signaling pathways.

In our study the immunoreactivity of the WNT5A protein was observed not only in the cytoplasm but also in the nuclei of EC cells and, to a lesser extent, in some epithelial cells of the control endometrial tissues. Interestingly, $W N T 5 A$ transcription can result in the synthesis of two different isoforms, long (380 amino-acids; The Consensus CDS accession number: CCDS46850) and short (365 amino-acids; CCDS58835) [25] that could be characterized by distinct biological activity [13]. The results of in silico analysis of their amino-acid sequences using nuclear localization signals (NLS) mapper [26] recognized a single putative NLS within the N-terminus of long WNT5A isoform and lack of it in the short isoform. Basing on NLS prediction it could be assumed that localization of WNT5A immunoreactivity within the cell may differ depending on the type of expressed 
isoform, with long isoform being directed both to the cytoplasm and nucleus and short isoform expressed exclusively in the cytoplasm [26]. Therefore, we suggest that the changes in the subcellular localization of WNT5A immunoreactivity are associated with the altered expression pattern of WNT5A isoforms as it was demonstrated in other human cancers such as cervical cancer, breast cancer and neuroblastoma [13]. The QPCR assay used in our study did not discriminate between the mRNA variants encoding for long and short WNT5A isoforms. In our immunohistochemical study, we could detect both WNT5A protein isoforms, since the applied primary antibody recognizes $23-80$ aa of the long isoform as well as 15-72 aa of the short WNT5A isoform, respectively. Thus, further studies aiming to determine the expression of WNT5A isoforms in EC samples could clarify whether the possible role of this protein in EC pathogenesis is isoform-specific. Furthermore, our results showed that WNT5A mRNA correlated negatively with cytoplasmic and positively with nuclear WNT5A immunoreactivity levels in EC cells. We believe that the latter observation could be, at least partially, also elucidated by the analysis of WNT5A expression with respect to the expressed variants of transcript or isoforms.

Our study revealed that $W N T 5 A$ mRNA and immunoreactivity levels correlated with preoperative counts of white blood cells and MPV. Since the correlations with granulocytes and lymphocytes count were found for the cytoplasmic but not nuclear immunoexpression of WNT5A, its role as secretory molecule and modulator of immune processes can be considered [15]. Other authors have shown that biomarkers of inflammation such as elevated neutrophil to lymphocyte ratio or platelet to lymphocyte ratio are significant prognostic factors in EC and correlate with worse prognosis [27]. In our study the lower cytoplasmic immunoexpression of WNT5A was positively associated with lymphocyte and negatively with neutrophils counts. This relationships could indicate the existence of direct or indirect links between the WNT5A expressed in endometrial tumor and inflammatory processes. Indeed, WNT5A protein have been considered tumor-derived immunomodulator that has been shown to influence chemotactic cell migration in ovarian cancer cell line SKOV-3 [28] and promote differentiation of regulatory $\mathrm{T}$-cells by activation of dendritic cells in melanoma [29]. Interestingly, it was demonstrated that WNT5A potentiated thromboxane A2 mimetic-induced platelet aggregation and therefore the secretion of this protein by circulating cancer cells might promote metastatic progression suggesting that WNT5A can promote tumor progression by its interaction with the platelets [30]. It cannot be excluded that in EC, WNT5A has analogous functions to those reported in the aforementioned studies [28-30]. However, further experiments are needed to evaluate its importance in establishing of tumor microenvironment and blood-borne metastasis.

In conclusion, the investigations of the WNT5A expression in the endometrial cancer suggest that this signaling molecule could function in the development of this malignancy as tumor suppressor and/or modulator of immune response and could be considered as a potential marker in EC. However, the precise role of WNT5A in the pathogenesis of endometrial cancer must be elucidated in further experiments.

\section{Acknowledgments}

The research was financed from statutory grant (61.610.001-300) of the School of Medicine, Collegium Medicum, University of Warmia and Mazury, Olsztyn, Poland.

\section{Disclosure Statement}

The authors have no conflicts of interest to declare.

\section{References}

1. Ferlay J, Soerjomataram I, Dikshit R, et al. Cancer incidence and mortality worldwide: sources, methods and major patterns in GLOBOCAN 2012. Int J Cancer. 2015; 136(5): E359-E386, doi: 10.1002/ijc.29210, indexed in Pubmed: 25220842.

2. Kaaks R, Lukanova A, Kurzer MS. Obesity, endogenous hormones, and endometrial cancer risk: a synthetic review. Cancer Epidemiol Biomarkers Prev 2002;11:1531-43. PMID: 12496040.

3. Renehan AG, Tyson M, Egger M, et al. Body-mass index and incidence of cancer: a systematic review and meta-analysis of prospective observational studies. Lancet. 2008; 371(9612): 569-578, doi: 10.1016/S0140-6736(08)60269-X, indexed in Pubmed: 18280327.

4. Siegel RL, Fedewa SA, Miller KD, et al. Cancer statistics, 2015. CA Cancer J Clin. 2015; 65(1): 5-29, doi: 10.3322/ caac.21254, indexed in Pubmed: 25559415.

5. Bokhman JV. Two pathogenetic types of endometrial carcinoma. Gynecol Oncol. 1983; 15(1): 10-17, indexed in Pubmed: 6822361.

6. Ryan AJ, Susil B, Jobling TW, et al. Endometrial cancer. Cell Tissue Res. 2005; 322(1): 53-61, doi: 10.1007/s00441005-1109-5, indexed in Pubmed: 15947972.

7. Santacana M, Maiques O, Valls J, et al. A 9-protein biomarker molecular signature for predicting histologic type in endometrial carcinoma by immunohistochemistry. Hum Pathol. 2014; 45(12): 2394-2403, doi: 10.1016/j.humpath.2014.06.031, indexed in Pubmed: 25282036.

8. Coenegrachts L, Garcia-Dios DA, Depreeuw J, et al. Mutation profile and clinical outcome of mixed endometrioid-serous endometrial carcinomas are different from that of pure endometrioid or serous carcinomas. Virchows Arch. 2015; 466(4): 415-422, doi: 10.1007/s00428-015-1728-5, indexed in Pubmed: 25677978.

9. Hoang LN, McConechy MK, Köbel M, et al. Histotype-genotype correlation in 36 high-grade endometrial carcinomas. 
Am J Surg Pathol. 2013; 37(9): 1421-1432, doi: 10.1097/ PAS.0b013e31828c63ed, indexed in Pubmed: 24076778.

10. Krejczy K, Cymbaluk-Płoska A, Kwiatkowski S, et al. Molecular characteristics of endometrial cancer and their potential effect on clinical management. Current Gynecologic Oncology. 2019; 16(4): 245-250, doi: 10.15557/cgo.2018.0028.

11. Nusse R, Clevers H. Wnt/ -Catenin Signaling, Disease, and Emerging Therapeutic Modalities. Cell. 2017; 169(6): 985-999, doi: 10.1016/j.cell.2017.05.016, indexed in Pubmed: 28575679.

12. Yamaguchi TP, Bradley A, McMahon AP, et al. A Wnt5a pathway underlies outgrowth of multiple structures in the vertebrate embryo. Development. 1999; 126(6): 1211-1223, indexed in Pubmed: 10021340.

13. Bauer M, Bénard J, Gaasterland T, et al. WNT5A encodes two isoforms with distinct functions in cancers. PLoS One. 2013; 8(11): e80526, doi: 10.1371/journal.pone.0080526, indexed in Pubmed: 24260410.

14. McDonald SL, Silver A. The opposing roles of Wnt-5a in cancer. Br J Cancer. 2009; 101(2): 209-214, doi: 10.1038/ sj.bjc.6605174, indexed in Pubmed: 19603030.

15. Asem MS, Buechler S, Wates RB, et al. Wnt5a Signaling in Cancer. Cancers (Basel). 2016; 8(9), doi: 10.3390/cancers8090079, indexed in Pubmed: 27571105.

16. Bui TD, Zhang L, Rees MC, et al. Expression and hormone regulation of Wnt2, 3, 4, 5a, 7a, 7b and 10b in normal human endometrium and endometrial carcinoma. Br J Cancer. 1997; 75(8): 11311136, doi: 10.1038/bjc.1997.195, indexed in Pubmed: 9099960.

17. Greene FL, Page DL, Fleming ID, et al. Gynecological sites. In: American Joint Committee on Cancer: AJCC Cancer Staging Manual. 6th edition. Springer, New York, NY; 2002: 241-300.

18. Petru E, Lück HJ, Stuart G, et al. Gynecologic Cancer Intergroup (GCIG). Gynecologic Cancer Intergroup (GCIG) proposals for changes of the current FIGO staging system. Eur J Obstet Gynecol Reprod Biol. 2009; 143(2): 69-74, doi: 10.1016/j.ejogrb.2008.12.015, indexed in Pubmed: 19195765.

19. Livak KJ, Schmittgen TD. Analysis of relative gene expression data using real-time quantitative PCR and the 2(-Delta Delta C(T)) Method. Methods. 2001; 25(4): 402-408, doi: 10.1006/ meth.2001.1262, indexed in Pubmed: 11846609.

20. Romani C, Calza S, Todeschini P, et al. Identification of optimal reference genes for gene expression normalization in a wide cohort of endometrioid endometrial carcinoma tissues. PLoS One. 2014; 9(12): e113781, doi: 10.1371/journal. pone.0113781, indexed in Pubmed: 25473950.
21. Remmele W, Stegner HE. [Recommendation for uniform definition of an immunoreactive score (IRS) for immunohistochemical estrogen receptor detection (ER-ICA) in breast cancer tissue]. Pathologe. 1987; 8(3): 138-140, indexed in Pubmed: 3303008.

22. Bitler BG, Nicodemus JP, Li H, et al. Wnt5a suppresses epithelial ovarian cancer by promoting cellular senescence. Cancer Res. 2011; 71(19): 6184-6194, doi: 10.1158/0008-5472. CAN-11-1341, indexed in Pubmed: 21816908.

23. Peng $\mathrm{C}$, Zhang $\mathrm{X}, \mathrm{Yu} \mathrm{H}$, et al. Wnt5a as a predictor in poor clinical outcome of patients and a mediator in chemoresistance of ovarian cancer. Int J Gynecol Cancer. 2011; 21(2): 280-288, doi: 10.1097/IGC.0b013e31820aaadb, indexed in Pubmed: 21270611.

24. Lin $\mathrm{Li}$, Liu Y, Zhao W, et al. Wnt5A expression is associated with the tumor metastasis and clinical survival in cervical cancer. Int J Clin Exp Pathol. 2014; 7(9): 6072-6078, indexed in Pubmed: 25337253.

25. Farrell CM, O'Leary NA, Harte RA, et al. Current status and new features of the Consensus Coding Sequence database. Nucleic Acids Res. 2014; 42(Database issue): D865-D872, doi: 10.1093/nar/gkt1059, indexed in Pubmed: 24217909.

26. Kosugi S, Hasebe M, Tomita M, et al. Systematic identification of cell cycle-dependent yeast nucleocytoplasmic shuttling proteins by prediction of composite motifs. Proc Natl Acad Sci U S A. 2009; 106(25): 10171-10176, doi: 10.1073/ pnas.0900604106, indexed in Pubmed: 19520826.

27. Cummings M, Merone L, Keeble C, et al. Preoperative neutrophil:lymphocyte and platelet:lymphocyte ratios predict endometrial cancer survival. Br J Cancer. 2015; 113(2): 311-320, doi: 10.1038/bjc.2015.200, indexed in Pubmed: 26079303.

28. Arabzadeh S, Hossein G, Zarnani AH. Wnt5A exerts immunomodulatory activity in the human ovarian cancer cell line SKOV-3. Cell Biol Int. 2016; 40(2): 177-187, doi: 10.1002/ cbin.10551, indexed in Pubmed: 26462870.

29. Holtzhausen A, Zhao F, Evans KS, et al. Melanoma-Derived Wnt5a Promotes Local Dendritic-Cell Expression of IDO and Immunotolerance: Opportunities for Pharmacologic Enhancement of Immunotherapy. Cancer Immunol Res. 2015; 3(9): 1082-1095, doi: 10.1158/2326-6066.CIR-14-0167, indexed in Pubmed: 26041736.

30. Kim SY, Kim S, Yun-Choi HS, et al. Wnt5a potentiates U46619-induced platelet aggregation via the PI3K/Akt pathway. Mol Cells. 2011; 32(4):333-336, doi: 10.1007/s10059-0110134-3, indexed in Pubmed: 21870110.

Submitted: 17 May, 2019

Accepted after reviews: 21 May, 2019 Available as AoP: 14 June, 2019 